\title{
Common Coding of Observation and Execution of Action in 9-Month-Old Infants
}

\author{
Matthew R. Longo and Bennett I. Bertenthal \\ Department of Psychology \\ University of Chicago
}

\begin{abstract}
Do 9-month-old infants motorically simulate actions they perceive others perform? Two experiments tested whether action observation, like overt reaching, is sufficient to elicit the Piagetian A-not-B error. Infants recovered a toy hidden at location A or observed an experimenter recover the toy. After the toy was hidden at location B, infants in both conditions perseverated in reaching to $\mathrm{A}$, demonstrating that active search by the infant is not necessary for the A-not-B error. Consistent with prior research, infants displayed an ipsilateral bias when reaching, the so-called mysterious midline barrier. A similar ipsilateral bias was also observed depending on the manner in which the experimenter reached; infants perseverated following observation of ipsi- but not contralateral reaches by the experimenter. Thus, infants perseverated only following observation of actions they themselves were able to perform, suggesting that they coded others' actions in terms of motor simulation.
\end{abstract}

The ability to represent and understand the behaviors of others is crucial for interacting effectively in our social world. Much of this understanding takes place with little awareness of the perceived actions or responses that are involved. When observing the behaviors of another, we tend to implicitly know their wants, desires, and intentions (Blakemore \& Decety, 2001). Moreover, our responses are automatically shaped by these behaviors (Blakemore \& Frith, 2005). Dating back at least to Darwin's (1872/1965) observations of spectators at sporting events, researchers have commonly noted that people will automatically imitate actions they observe others perform. For example, we direct our gaze in the same direction we see another individual look (Driver et al., 1999), and we mimic the facial

Correspondence should be addressed to Matthew R. Longo, Department of Psychology, University of Chicago, 5848 South University Avenue, Chicago, IL 60637. E-mail: mlongo@ uchicago.edu 
expression or gestures shown by someone else (for review see Dijksterhuis \& Bargh, 2001). This automatic perception-action link contributes to the social resonance by which we coordinate and cooperate with each other and form affiliations or aversions.

One intriguing interpretation for these effects is that the perception of actions automatically activates corresponding motor programs in the perceiver. This proposal emerged originally from James's (1890) ideomotor theory and from Dromard's (1905) clinical observations of echopraxia, and was elaborated more recently in Prinz's (1990) common coding framework. In essence, this framework suggests that the representation of a perceived action involves simulative production of that action on the part of the observer. This covert motor activation results in the observation of an action facilitating its execution.

In recent years, a number of convergent lines of research have reported findings supporting this simulative theory of action perception. Rizzolatti and colleagues (di Pellegrino, Fadiga, Fogassi, Gallese, \& Rizzolatti, 1992; Rizzolatti, Fadiga, Gallese, \& Fogassi, 1996), for example, described a class of so-called mirror neurons in the premotor cortex of macaques that respond both when the monkey performs a specific action and when the monkey passively observes the experimenter perform that same action. These findings provide evidence that the observation and execution of action share a common neural representation. Recent results from electrophysiological (e.g., Fadiga, Fogassi, Pavesi, \& Rizzolatti, 1995), neuroimaging (e.g., Iacoboni et al., 1999), and behavioral (e.g., Bertenthal, Longo, \& Kosobud, in press; Brass, Bekkering, Wohlschläger, \& Prinz, 2000) studies suggest that a similar observation-execution matching system is neurally represented in the brains of humans (see Rizzolatti \& Craighero, 2004, for a review).

Thus far, we know little about the functioning of an observation-execution matching system in human infants. One recent exception is the work of Sommerville and Woodward (2005), who found that the emerging ability of 10month-old infants to solve a means-ends task was predictive of their perception of another agent performing a similar means-ends task. This result suggests a possible link between the perception and production of actions in infancy; only infants who could perform the means-ends sequence perceived such behavior on the part of the experimenter as goal-directed. In a subsequent study, Sommerville, Woodward, and Needham (2005) examined the effects of manipulating action experience on 3-month-old infants' perception of an experimenter's action. Providing these prereaching infants with Velcro mittens enabled them to directly experience the effects of reaching, and this brief experience led them to perceive the actions of the experimenter as goal-directed in a habituation task. By contrast, infants who were tested in the habituation procedure before the mittens task showed no such effect. Thus, early experience with reaching for objects altered infants' perception of the actions of others, but the converse was not true. 
Although the preceding evidence is suggestive of a functioning observationexecution matching system in infants, it is also necessary to show that the relation between the perception and production of actions is bidirectional, and more specifically that the perception of actions facilitates the execution of these actions in the observer. The facilitation of actions following their observation is a central finding in the literature on the human mirror system. Indeed, a number of authors (e.g., Jeannerod, 1994; Rizzolatti \& Craighero, 2004) theorize that a major function of an observation-execution matching system is to facilitate both imitation and action understanding via the observation of actions. The observation of these actions elicits in the perceiver offline motor simulations of these same actions. The studies reported here were designed to complement previous studies showing a relation between the production and perception of actions by investigating the effect of action perception on action production in 9-month-old infants, using a variant of the A-not-B task.

\section{The A-Not-B Error}

The Piagetian A-not-B error, shown by 8- to 12-month-old infants, is among the most consistently replicable findings in developmental psychology. In this task, infants first search correctly for an object they see hidden in one location (A) on one or more trials, but then continue to search at the A location after the object has been hidden in a new location (B). According to Piaget (1937/1954), the error arises because the infant conceives of the object as a thing to be found at the location where it was previously found, regardless of where it is observed to be hidden. More recent research has extended Piaget's findings, demonstrating that infants will make perseverative reaching errors even if transparent covers are used to hide the objects (Butterworth, 1977; Harris, 1974; Sophian \& Yengo, 1985; although see Yates \& Bremner, 1988), if the objects are unhidden (Bremner \& Knowles, 1984; Butterworth, 1977), if a different object is hidden on the B trials (Evans \& Gratch, 1972), and even if no objects are hidden at all (Munakata, 1997; Smith, Thelen, Titzer, \& McLin, 1999)!

Recent accounts of the error emphasize the role of repeated reaching to the A location. Diamond (1985, 1991), for example, argued that two processes limit infants' abilities to succeed on the A-not-B task: working memory and the ability to inhibit a rewarded motor response to A. Smith, Thelen, and colleagues (Smith et al., 1999; Thelen, Schoner, Scheier, \& Smith, 2001) claim that the error arises from the task dynamics of reaching, such that "[i]nfants make perseverative location errors because the motor memory of one reach persists and influences subsequent reaches" (Thelen et al., 2001, p. 9). Zelazo and colleagues (Marcovitch \& Zelazo, 1999; Zelazo, Reznick, \& Spinazzola, 1998) account for perseverative responses in young children in terms of the relative dominance of a responsebased system "activated by motor experience" over a conscious representational 
system (Marcovitch \& Zelazo, 1999, p. 1308). In each of these accounts, a history of reaches to the A location is a crucial aspect of the perseverative response.

Previous research investigating the observation of search behaviors on subsequent performance in the A-not-B error is equivocal. Landers (1971) investigated the role of reaching in the A-not-B error, finding that infants who observed him find a toy six to eight times before searching twice at A did not make more errors than infants who had not had such observational experience. Landers interpreted these results as evidence that "simply watching the experimenter hide and uncover a toy at A does not establish the A side as a 'special' place, while active search does" (p. 53). Although these results seem to support the necessity of active search, they are inconclusive because infants in all conditions searched at least twice at A before they were tested at B. Conceivably, two trials of active search were sufficient to produce a perseverative error regardless of whether or not these two trials were preceded by six observation trials.

In contrast to the preceding study, two dissertations (Diamond, 1983; Evans, 1973) reported that infants will make A-not-B errors even if they have only observed the experimenter reach to the A location. Although suggestive, both of these studies suffer from methodological problems, making them difficult to interpret. Evans's (1973) study, for example, relied on a finding of no difference between conditions, making these results difficult to interpret because they were based on demonstrating a null hypothesis. Diamond (1983) did compare performance on B trials in both observation and reaching conditions to a baseline, but, as the author acknowledged, this baseline is ambiguous as a comparison for the observation trials.

Two recent studies of perseverative search in toddlers also examined active search versus passive observation. Zelazo et al. (1998) found that 24-month-olds perseverated in searching in one location after recovering an object from that location, but not after seeing the experimenter recover the object. They concluded that active search was required to elicit perseveration (but see the Discussion for an alternative interpretation). Spencer and Schutte (2004), however, using a continuous as opposed to discrete search task, found that 29-month-olds and 4-yearolds' searches for a hidden toy were biased in the direction toward which they had simply observed the experimenter recover the toy.

Thus, there is some ambiguity in the literature regarding the necessity of active reaching for the production of perseverative errors. In contrast to studies emphasizing the crucial role of active motor history in the A-not-B error, some preliminary reports suggest that observing another person reach is sufficient to elicit the error. By 2.5 years of age there is some evidence that such action observation results in perseveration. These findings are in seeming contradiction to theories of perseveration in infants and young children that give a central role to motor history. Yet, from the perspective of a common coding framework for the observation and execution of actions, the preceding contradiction may be more apparent than 
real. If infants represent observed behaviors simulatively, the passive perception of the experimenter reaching to the A location should activate similar motor representations in the infants' brains as if they had themselves reached. These studies sought to investigate whether infants would make the A-not-B error after merely watching someone else find a toy at the A location. Such perseveration would be consistent with offline simulation of perceived actions in infancy, and in conjunction with the previously cited research, provide evidence for the functioning of an observation-execution matching system in human infants.

\section{Ipsilateral Biases and the Mysterious Midline Barrier}

A tendency originally described by Head $(1920,1926)$ in his studies of aphasic patients may also be relevant to the interpretation of perseveration in this situation. Head presented his patients with so-called hand, eye, and ear tests in which he would stand before the patient and raise one hand to touch an eye or an ear. Head found that patients, when asked to imitate, frequently failed to cross the body midline, performing an ipsilateral movement when a contralateral movement was modeled. ${ }^{1}$ Although Head considered this error in adults to be a serious sign of neurological insult, such ipsilateral biases (or failures to cross the midline) have been observed in infancy as well as in older children. Bruner (1969), for example, referred to the apparent inability of young infants to reach across the body midline as the "mysterious midline barrier," arguing that contralateral reaches do not occur before 7 months of age. Subsequent studies using simpler tasks report contralateral reaches at younger ages, which, depending on the task, emerge anywhere between 4 (Provine \& Westerman, 1979) and 7 (Morange \& Bloch, 1996) months. Nevertheless, a clear preference for ipsilateral reaches is consistently observed in early development. Contralateral reaching becomes more frequent with age both on reaching tasks during infancy (van Hof, van der Kamp, \& Savelsbergh, 2002) and in hand, eye, and ear tasks in later childhood (Bekkering, Wohlschläger, \& Gattis, 2000; Schofield, 1976; Wapner \& Cirillo, 1968).

Such an ipsilateral bias may influence the likelihood of the infant covertly imitating the experimenter. A number of recent studies suggest that simulation by the mirror system is limited to those actions within the motor repertoire of the observer (e.g., Calvo-Merino, Glaser, Grèzes, Passingham, \& Haggard, 2005; Longo, Kosobud, \& Bertenthal, 2005). If an observer is unable to perform an action, he or she cannot simulate it, and hence, will not automatically imitate it. Because infants have difficulty reaching contralaterally, simulation of observed contralateral reaches should be weaker than that for ipsilateral reaches, or perhaps absent

\footnotetext{
${ }^{1}$ Head $(1920,1926)$ also observed another pattern of error in which patients would respond using the hand opposite that used by the experimenter, as if facing themselves in a mirror. Head termed this pattern reversal.
} 
entirely. Thus, if observation-execution matching mechanisms are operative, not only should infants perseverate after observing the experimenter reach to A, but this tendency should be stronger following observation of ipsi- than of contralateral reaches.

\section{EXPERIMENT 1}

This experiment compared performance on observational and canonical versions of the A-not-B task. One group of infants was allowed to search on the A trials, and the other watched as the experimenter recovered the object on the A trials. Both groups were allowed to search on the B trials. If the infant's motor responses are unrelated to observing the experimenter reach, then accuracy on B trials following such observation should not differ from search performance on A trials in the standard condition. If, on the other hand, observing the experimenter's action activates similar representations as executing the same action, then successful search on B trials should be significantly poorer than search on A trials as reported in the canonical A-not-B paradigm.

One reason for past inconsistencies in the empirical literature is that procedures commonly used in administering the A-not-B task make it difficult to determine the exact history of reaches to A and, thus, the role of motor history in the error. Smith et al. (1999) cited three such procedures: (a) the use of pretraining trials at the A location, (b) continuing A trials until a criterion level of success is reached, and (c) the use of multiple reversal trials. Each of these three concerns was addressed in this experiment: (a) Pretraining trials were conducted using a single-well apparatus; (b) no criterion of success was required before the toy was hidden at B, thus ensuring a constant number of A trials; and (c) only one set of $\mathrm{A}$ and $\mathrm{B}$ trials was used, meaning that multiple reversals were not an issue.

\section{Methods}

Participants. Forty 9-month-old infants participated in this experiment. Testing began on an additional 21 infants whose data were not included due to fussiness (4 infants), experimenter error ( 8 infants), reaching to both locations simultaneously ( 3 infants), or refusal to reach (6 infants). Twenty of the infants were assigned to a canonical reaching condition $(M=38.8$ weeks, range $=$ 36.4-41.0) and the other 20 to a looking condition $(M=38.6$ weeks, range $=$ 36.7-40.6). Children were recruited from a participant database maintained by the Center for Infant Studies at the University of Chicago.

Materials. The experimental apparatus (see Figure 1) consisted of a brown cardboard boxtop $(30.5 \times 40.6 \times 4.45 \mathrm{~cm})$ with two cylindrical wells $(12 \mathrm{~cm}$ 


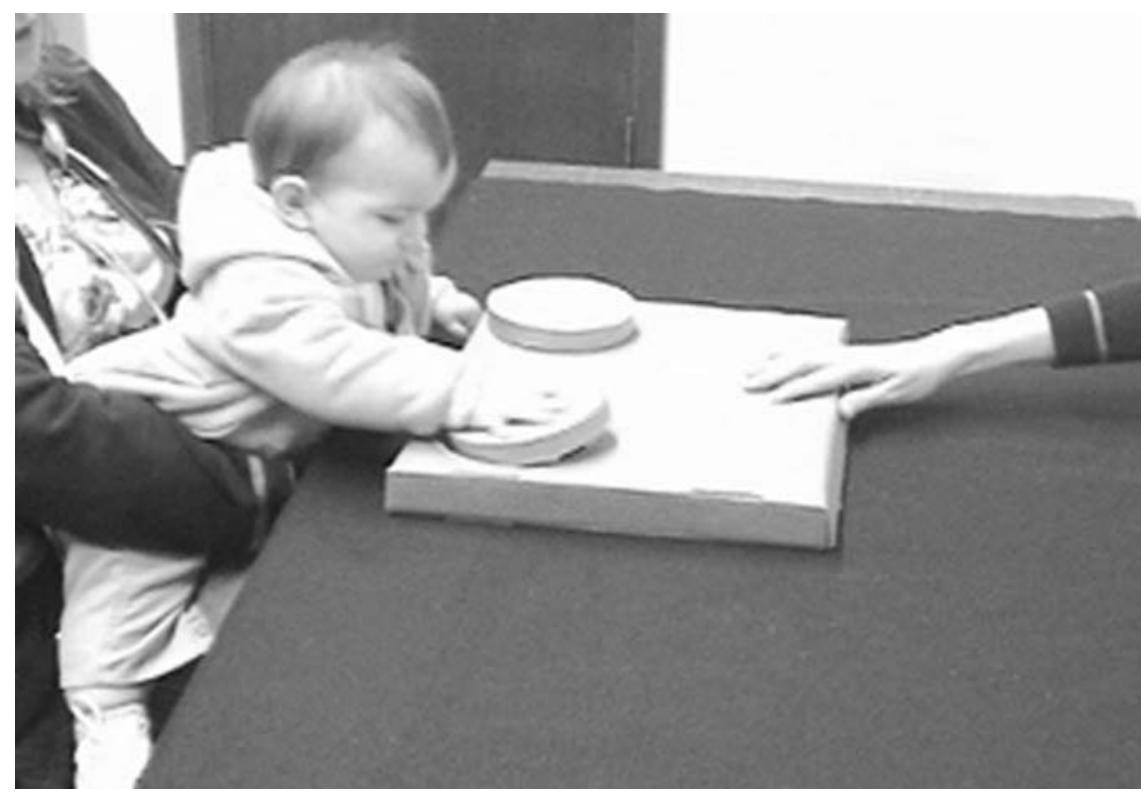

FIGURE 1 Infant searching for toy in one of the two hiding wells.

diameter) set into it. The perimeters of the wells were set $1 \mathrm{~cm}$ from the near edge of the box and the wells were spaced $12.7 \mathrm{~cm}$ from each other (nearest edge to edge). A similar apparatus with a single well in the center was used for training trials. Cylindrical lids (12.7 cm diameter, $2.5 \mathrm{~cm}$ height) were used to cover the wells.

Procedure. Infants were seated on their mother's lap in front of a table covered with black felt and allowed to play with a toy (a rattle or a plastic Big Bird) for several seconds. Four pretraining trials were administered using procedures similar to those used by Smith et al. (1999). On the first pretraining trial, the toy was placed on top of the single covered well. On the second trial, the toy was placed in the well but with one end sticking out. On the third trial, the toy was placed completely in the well but left uncovered. On the final trial, the toy was placed completely in the well and covered.

The experimental trials used the two-well apparatus and consisted of three A trials and one $\mathrm{B}$ trial. ${ }^{2}$ Infants in the reaching condition were allowed to search on

\footnotetext{
${ }^{2} \mathrm{Up}$ to four blocks of three A trials and a B trial were administered to each infant, but only the first of these was included in the analysis. Not all infants completed all blocks. On average, 2.9 blocks were collected per infant.
} 
all trials. Infants in the looking condition were only allowed to search on the B trial and observed the experimenter recover the object on the A trials. On each trial, the toy was waved and the infant's name was called to attract his or her attention. The experimenter removed the lid with one hand and placed the toy in the well with the other. A-location (right or left) and experimenter's arm (right or left; coded as which arm the experimenter used to hide the toy ${ }^{3}$ ) were counterbalanced between infants. Thus, the experimenter's reaches were ipsilateral half of the time (right-handed reach to the location on the right or left-handed reach to location on the left) and contralateral half the time (right-handed reach to the location on the left or left-handed reach to the location on the right). For A trials in the reaching condition, the apparatus was slid forward to within the baby's reach following a 3 -sec delay. If, after $10 \mathrm{sec}$, the infant had not retrieved the toy from the A location, the experimenter uncovered the well and encouraged the infant to retrieve the toy. For A trials in the looking condition, the experimenter recovered the toy following a 3 -sec delay. The experimenter used the same arm to retrieve the toy as was used to hide the toy. On B trials, in both conditions, the experimenter hid the toy (using the same hand as on the A trials) and then the apparatus was moved to within the infant's reach following a 3-sec delay.

\section{Results and Discussion}

The proportion of infants in the reaching condition making errors on the A trials was used as a baseline for comparisons with both types of B trials. These data are shown in Figure 2. Chi-square analyses were used to compare the proportion of infants making errors in each condition (see Figure 2). Because the three A trials were not independent of each other, separate analyses were conducted comparing performance on reaching and looking B trials to each A trial. Infants in the reaching condition were significantly more likely to make an error on B trials (15 of 20) than on any of the A trials (see Table 1 for statistics), consistent with the canonical A-not-B error. Infants in the looking condition also made significantly more errors on B trials (12 of 20) than infants in the reaching condition did on A trials (see Table 1 for statistics). The likelihood of making an error did not differ significantly between the two B conditions, $\chi^{2}(1, N=40)=1.03, n s$.

\footnotetext{
${ }^{3}$ Although both hands are used for reaching, there are at least two main reasons why we believe the hand used for hiding and recovering the toy is more salient than the hand used for uncovering. First, during hiding, the toy is being waved and the infants' attention is directed to the hiding hand at the same time that the cover is being removed by the other hand. Second, the hand used to recover the object should be more salient than the hand used to uncover the lid simply because it is directed at the toy, which was the likely focus of infants' attention. Relevant to this interpretation, 12-month-old infants interpret uncovering a container as an intermediate stage in a means-ends sequence directed at recovering the object (Woodward \& Sommerville, 2000).
} 


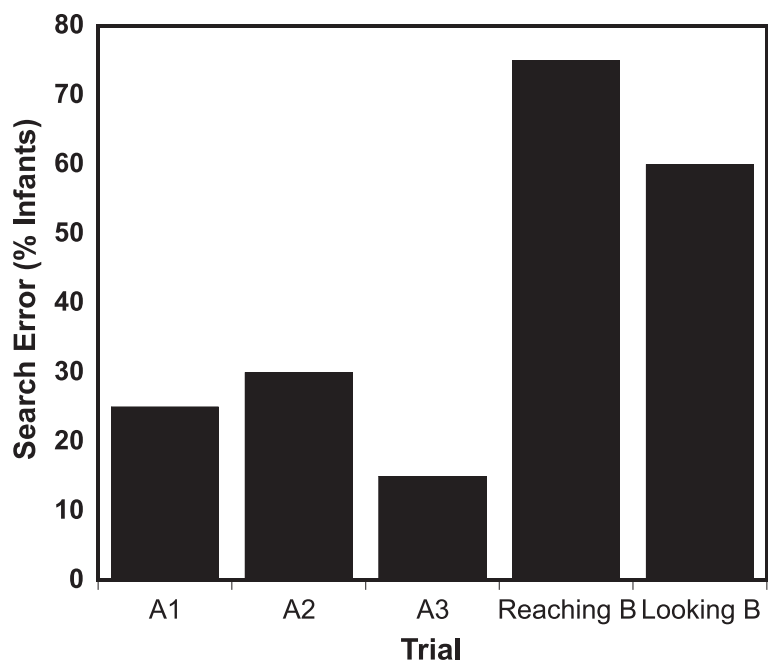

FIGURE 2 Percentage of infants searching incorrectly on first, second, and third reaching A trials, reaching B trial and looking B trial.

TABLE 1

Comparisons Between Search Errors on the Three Reaching A Trials and the Reaching and Looking B Trials

\begin{tabular}{lllr}
\hline B Trial Type & A Trial & Test Statistic $^{a}$ & $p$ \\
\hline Reaching (15 of 20) & A1 (5 of 20) & McNemar's $\chi^{2}=8.33$ & $<.01$ \\
& A2 (6 of 20) & McNemar's $\chi^{2}=5.40$ & $<.05$ \\
Looking (12 of 20) & A3 (3 of 20) & McNemar's $\chi^{2}=9.00$ & $<.01$ \\
& A1 (5 of 20) & $\chi^{2}=5.01$ & $<.05$ \\
& A2 (6 of 20) & $\chi^{2}=3.64$ & .056 \\
& A3 (3 of 20) & $\chi^{2}=8.64$ & $<.01$ \\
\hline
\end{tabular}

Note. Numbers in parentheses indicate the number of infants making errors.

${ }^{\mathrm{a}}$ For the reaching B trials, McNemar's chi-squares for repeated measures were used.

These data suggest that looking A trials influenced performance on the B trials. Nonetheless, to establish that these responses are truly perseverative, as opposed to simply random, it is necessary to demonstrate errors on significantly more than $50 \%$ of the trials. Binomial tests revealed greater than chance perseveration on reaching $(p<.05)$, but not on looking B trials $(p>.1)$. Thus, these data provide 
strong evidence that infants perseverate after reaching A trials, but the evidence of perseveration following looking A trials is equivocal.

A second analysis revealed that infants showed an ipsilateral bias in their reaching. On looking B trials, $90 \%$ (18 of 20) of the infants made ipsilateral reaches, significantly more than would be expected by chance $(p<.001$, binomial test). Similar ipsilateral biases were observed on the three reaching A trials $(81.7 \%), t(19)=4.79$, $p<.001$, and the reaching B trials $\left(73.7 \%, 14\right.$ of 19 one-handed reaches ${ }^{4} ; p<.05$, binomial test). Intriguingly, infants' perception of the experimenter's actions mirrored their motor bias, as infants in the looking condition were more likely to reach to location A than to location B when the experimenter had reached ipsilaterally ( 8 of 10), rather than contralaterally ( 4 of 10), although this effect was only marginally significant, $\chi^{2}(1, N=20)=3.33, p=.068$ (see Figure 3). This result is suggestive that infants' responses following observation of the experimenter's reaches may not be random, but vary systematically with those of the experimenter.

\section{EXPERIMENT 2}

In the previous experiment, perseverative reaching was shown following reaching on the A trials, but perseverative reaching following observation of reaching was ambiguous as search performance on subsequent B trials did not differ significantly from chance. Marcovitch, Zelazo, and Schmuckler (2002) found that the likelihood of a perseverative search error increased as the number of A trials increased, at least between the range of one to six. ${ }^{5}$ If the A-not-B error is indeed a function of similar mechanisms inducing perseverative search in both the reaching and looking conditions, then we would expect the likelihood of search errors in the looking condition to also increase with the presentation of more A trials. Thus, to increase the likelihood of finding perseveration at greater than chance levels, the following experiment included six looking A trials instead of three. An additional goal was to provide further evidence for a difference in perseverative reaching depending on whether the experimenter reached ipsilaterally or contralaterally on the A trials.

\section{Methods}

Participants. Thirty 9-month-old infants participated in this study $(M=38.9$ weeks, range $=36.6-41.3$ ). Testing began on an additional 6 infants who were

\footnotetext{
${ }^{4}$ One infant in the reaching condition reached with both hands simultaneously on the B trial. This trial was excluded from the analysis of the ipsilateral bias.

${ }^{5}$ Intriguingly, Marcovitch et al. (2002) found significantly fewer A-not-B errors after 11 A trials than after 6 . They account for this U-shaped pattern in terms of a two-stage model wherein repeated reaches increase habit strength but also increase the likelihood of conscious reflection on the task.
} 
eliminated due to experimenter error ( 3 infants), failure to search ( 2 infants), or lack of attention (1 infant). Children were recruited from a participant database maintained by the Center for Infant Studies at the University of Chicago.

Materials. All materials were identical to Experiment 1.

Procedure. Three changes were made to the procedures followed in the first experiment. First, only the looking condition was used. Second, the experimenter hid and recovered the toy six times at the A location, instead of three times as in Experiment 1. Third, because no differences were observed in the first experiment as a function of which arm the experimenter used to hide the toy, the experimenter always used the right arm.

\section{Results and Discussion}

Seventy percent ( 21 of 30 ) of infants made the A-not-B error, significantly more than half of the sample ( $p<.05$, binomial test). This finding suggests that infants' reaches following observation of a reaching action are perseverative, not random. As in the first experiment, an ipsilateral bias in infants' reaching was observed, with $85 \%$ ( 23 of 27 ) of one-handed reaches ${ }^{6}$ scored as ipsilateral $(p<.0005$, binomial test). Furthermore, this ipsilateral bias translated to the infants' representation of the experimenter's reaching. Perseveration was observed when the experimenter had reached ipsilaterally on the A trials (13 of 15 infants made the error; $p<.01$, binomial test), but not when the experimenter had reached contralaterally ( 8 of 15 made the error; $n s$, binomial test). This difference between conditions was significant, $\chi^{2}(1, N=30)=3.97, p<.05$ (see Figure 3$)^{7}$

\section{GENERAL DISCUSSION}

There are two main findings that emerge from these experiments. The first is that 9-month-old infants make perseverative reaching (A-not-B) errors both following

\footnotetext{
${ }^{6}$ Three infants reached with both hands simultaneously. As in the first experiment, these trials were excluded from these analyses.

${ }^{7}$ One potential concern about this comparison between trials in which the experimenter reached ipsiversus contralaterally is the sample size. The chi-square test can yield biased results when small sample sizes are used. Agresti (1996) suggested that an average of at least five observations per cell is sufficient to avoid such problems. As this comparison involves a $2 \times 2$ table, there were five observations per cell in Experiment 1 and 7.5 in Experiment 2, both sufficiently large by Agresti's criteria. Nevertheless, the data were reanalyzed collapsing across experiments to increase the sample size. Significantly more perseveration was observed on trials when the experimenter's reach was ipsilateral than when it was contralateral, both using a chi-square test, $\chi^{2}(1, N=50)=7.22, p<.01$, and using the more conservative Fisher's exact test $(p<.02)$, which does not assume a minimum sample size.
} 


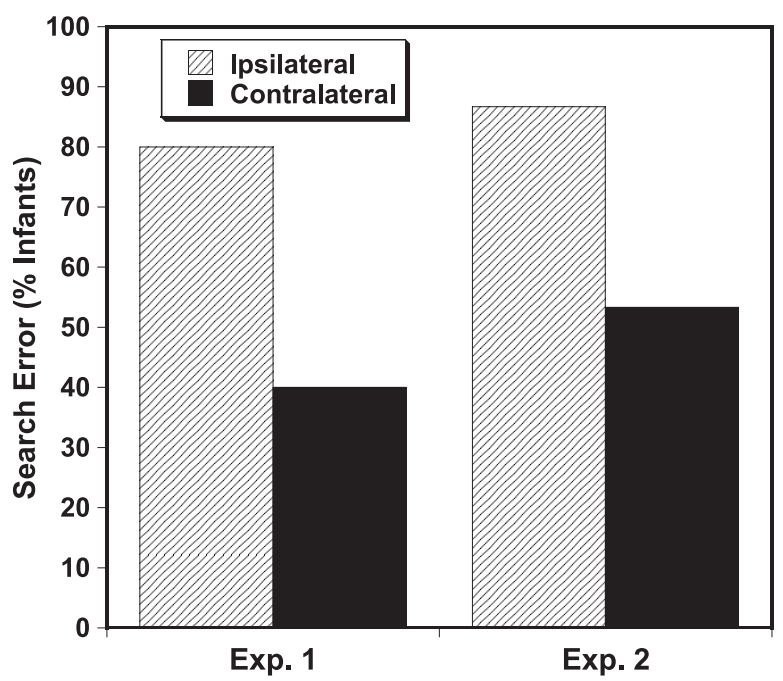

FIGURE 3 Percentage of infants searching incorrectly following observation of ipsi- and contralateral reaches by the experimenter in the looking condition of Experiment 1 and in Experiment 2.

active search at one location and following observation of active search by another person. During training, infants in the looking condition reached four times to a central location using the single-well apparatus, but never reached to the A location. Still, they were found to "perseverate" on their very first reach using the two-well apparatus. The second main finding was that an ipsilateral bias was observed both in infants' own reaching and in infants' perception of the experimeter's reach. Infants' perseveration was modulated by the manner of the experimenter's reach; infants perseverated after observing the experimenter reach ipsilaterally, but not contralaterally, mirroring the ipsilateral bias in infants' own reaching.

The preceding results reveal that perseveration in 9-month-old infants can result from the repetition of an action that has been observed as well as the repetition of an action that has been performed. Whereas prior research (e.g., Diedrich, Thelen, Smith, \& Corbetta, 2000; Smith et al., 1999) suggested that a history of active reaching is necessary to elicit a perseverative search error (Smith et al., 1999; Thelen et al., 2001), it now appears that this conclusion requires some amending. It is not active search, per se, that is necessary for inducing perseveration, but rather a history of motoric activation that is responsible for the perseverative error. If, as suggested in the introduction, infants represent perceived actions simulatively using a common representational code, perseverative errors in both 
reaching and looking conditions may result from residual activation of motor representations following reaching to the A location.

Can the results obtained in this study be explained by other mechanisms? Some researchers suggest that the crucial factor leading to search errors at the B location is not a history of reaching to the A location, but rather a history of attending to or planning to reach to the A location (e.g., Diedrich et al., 2000; Munakata, 1998; Ruffman \& Langman, 2002). A number of lines of research have shown performance on the A-not-B task to relate to infant attention. Studies coding the amount of time looking at each location (e.g., Bai \& Bertenthal, 1992; Horobin \& Acredolo, 1986; Landers, 1971) have found that increased attention to the A location is highly related to where infants reach. Similarly, altering the distinctiveness of the locations (Butterworth, Jarrett, \& Hicks, 1982; Diedrich, Highlands, Spahr, Thelen, \& Smith, 2001), adding (Munakata, 1997) or changing the salience of (Schuberth, Werner, \& Lipsitt, 1978) a hidden object, or explicitly drawing attention to one location (Smith et al., 1999) all influence the likelihood of perseveration.

In these experiments, greater attention to one location than the other will likely covary with the history of simulated reaching to that location, and thus it is difficult to disambiguate these two interpretations. Although a definitive answer concerning the relative contributions of attention and motor simulation must await further testing, at the very least the current evidence appears to challenge the sufficiency of an attentional explanation. In particular, it is not at all apparent how such an account would explain why infants showed greater perseveration after observing the experimenter reach ipsilaterally than contralaterally. Other potential explanations involving, for example, object representations, have similar difficulty accounting for this effect. By contrast, a common coding interpretation accounts for this effect in terms of infants' own difficulties with contralateral reaching, which should lead to weaker or absent motor simulation following observed contralateral, compared with ipsilateral reaches, and consequently less perseveration.

A common coding interpretation can also account for the apparent discrepancy between the perseverative responses observed in this study and the previously described study by Spencer and Schutte (2004) and the lack of perseveration observed by Zelazo et al. (1998). In the Spencer and Schutte study, as in this study, infants observed the experimenter reach only to the A location. In Zelazo et al.'s study, children observed the experimenter reach to every location, placing candy in the A location and holding up an empty bag at the other two locations. If, as hypothesized, perseveration in this study and that of Spencer and Schutte arises from simulative representation of the experimenter's reach, participants in Zelazo et al.'s study, who observed the experimenter reach to every location, would not be expected to perseverate to A because each location would have been represented.

In sum, perseverative reaching in 9-month-old infants occurs whether the infant searches actively or observes someone else reach. These results demonstrate an 
influence of action observation on action production, complementing the findings of Sommerville and colleagues (Sommerville \& Woodward, 2005; Sommerville et al., 2005) who observed effects of action production on action perception. Such a bidirectional influence is to be expected if a common representational system underlies both the perception and the production of action. This suggests that infants, like adult humans and monkeys, represent perceived actions by simulating their execution, and that the human mirror system may be functional by 9 months of age. Nine months, of course, does not necessarily represent a lower bound on the development of this mechanism. In fact, we speculate elsewhere that a common coding mechanism could explain the mimicry of facial gestures at even earlier ages (Longo \& Bertenthal, 2004).

\section{ACKNOWLEDGMENTS}

Portions of the data were previously presented at the biennial conference of the Society for Research in Child Development, Tampa, Florida, April 2003. This research was supported by a grant from the Robert McCormick-Tribune Foundation to Bennett I. Bertenthal and a predoctoral fellowship from the National Science Foundation to Matthew R. Longo. We would like to thank Sarah Kenny for assistance in data collection; Ross Kessler and Tanya Tang for assistance coding; and Richard Aslin, Janellen Huttenlocher, Amanda Woodward, and the anonymous reviewers for helpful discussion and comments on earlier versions of the article.

\section{REFERENCES}

Agresti, A. (1996). An introduction to categorical data analysis. New York: Wiley.

Bai, D. L., \& Bertenthal, B. I. (1992). Locomotor status and the development of spatial search skills. Child Development, 63, 215-226.

Bekkering, H., Wohlschläger, A., \& Gattis, M. (2000). Imitation of gestures in children is goal-directed. Quarterly Journal of Experimental Psychology, 53A, 153-164.

Bertenthal, B. I., Longo, M. R., \& Kosobud, A. (in press). Imitative response tendencies following observation of intransitive actions. Journal of Experimental Psychology: Human Perception and Performance.

Blakemore, S.-J., \& Decety, J. (2001). From the perception of action to the understanding of intention. Nature Reviews Neuroscience, 2, 561-567.

Blakemore, S.-J., \& Frith, C. (2005). The role of motor contagion in the prediction of action. Neuropsychologia, 43, 260-267.

Brass, M., Bekkering, H., Wohlschläger, A., \& Prinz, W. (2000). Compatibility between observed and executed finger movements: Comparing symbolic, spatial, and imitative cues. Brain and Cognition, 44, 124-143.

Bremner, J. G., \& Knowles, L. S. (1984). Piagetian stage IV search errors with an object that is directly accessible both visually and manually. Perception, 13, 307-314. 
Bruner, J. S. (1969). Eye, hand, and mind. In D. Elkind \& J. H. Flavell (Eds.), Studies in cognitive development: Essays in honor of Jean Piaget (pp. 223-235). Oxford, UK: Oxford University Press.

Butterworth, G. (1977). Object disappearance and error in Piaget's stage IV task. Journal of Experimental Child Psychology, 23, 391-401.

Butterworth, G., Jarrett, N., \& Hicks, L. (1982). Spatiotemporal identity in infancy: Perceptual competence or conceptual deficit? Developmental Psychology, 18, 435-449.

Calvo-Merino, B., Glaser, D. E., Grèzes, J., Passingham, R. E., \& Haggard, P. (2005). Action observation and acquired motor skills: An fMRI study with expert dancers. Cerebral Cortex, 15, $1243-1249$.

Darwin, C. (1965). The expression of the emotions in man and animals. Chicago: University of Chicago Press. (Original work published 1872)

Diamond, A. (1983). Behavior changes between 6 to 12 months of age: What can they tell us about how the mind of the infant is changing? Unpublished doctoral dissertation, Harvard University, Cambridge, MA.

Diamond, A. (1985). The development of the ability to use recall to guide action, as indicated by infants' performance on A-not-B. Child Development, 56, 868-883.

Diamond, A. (1991). Neuropsychological insights into the meaning of object concept development. In S. Carey \& R. Gelman (Eds.), The epigenesis of mind: Essays on biology and cognition (pp. 66-110). Hillsdale, NJ: Lawrence Erlbaum Associates, Inc.

Diedrich, F. J., Highlands, T. M., Spahr, K. A., Thelen, E., \& Smith, L. B. (2001). The role of target distinctiveness in infant perseverative reaching. Journal of Experimental Child Psychology, 78, 263-290.

Diedrich, F. J., Thelen, E., Smith, L. B., \& Corbetta, D. (2000). Motor memory is a factor in infant perseverative errors. Developmental Science, 3, 479-494.

Dijksterhuis, A., \& Bargh, J. A. (2001). The perception-behavior expressway: Automatic effects of social perception on social behavior. Advances in Experimental Social Psychology, 33, 1-39.

di Pellegrino, G., Fadiga, L., Fogassi, L., Gallese, V., \& Rizzolatti, G. (1992). Understanding motor events: A neurophysiological study. Experimental Brain Research, 91, 176-180.

Driver, J., Davis, G., Ricciardelli, P., Kidd, P., Maxwell, E., \& Baron-Cohen, S. (1999). Gaze perception triggers reflexive visuospatial orienting. Visual Cognition, 6, 509-540.

Dromard, G. (1905). Etude psychologique et clinique sur l'echopraxie [A psychological and clinical study of echopraxia]. Journal de Psychologie Normale et Pathologique, 2, 385-403.

Evans, W. F. (1973). The stage IV error in Piaget's theory of object concept development: An investigation of the role of activity. Unpublished doctoral dissertation, University of Houston, Houston, TX.

Evans, W. F., \& Gratch, G. (1972). The stage IV error in Piaget's theory of object concept development: Difficulties in object conceptualization or spatial localization? Child Development, 43, 682-688.

Fadiga, L., Fogassi, L., Pavesi, G., \& Rizzolatti, G. (1995). Motor facilitation during action observation: A magnetic stimulation study. Journal of Neurophysiology, 73, 2608-2611.

Harris, P. L. (1974). Perseverative search at a visibly empty place by young infants. Journal of Experimental Child Psychology, 18, 535-542.

Head, H. (1920). Aphasia and kindred disorders of speech. Brain, 43, 87-165.

Head, H. (1926). Aphasia and kindred disorders of speech. New York: Macmillan.

Horobin, K., \& Acredolo, L. (1986). The role of attentiveness, mobility history, and separation of hiding sites on stage IV search behavior. Journal of Experimental Child Psychology, 41, 114-127.

Iacoboni, M., Woods, R. P., Brass, M., Bekkering, H., Mazziotta, J. C., \& Rizzolatti, G. (1999). Cortical mechanisms of human imitation. Science, 286, 2526-2528.

James, W. (1890). The principles of psychology. New York: Dover.

Jeannerod, M. (1994). The representing brain: Neural correlates of motor intention and imagery. Behavioral and Brain Sciences, 17, 187-245. 
Landers, W. F. (1971). Effects of differential experience on infants' performance in a Piagetian stage IV object-concept task. Developmental Psychology, 5, 48-54.

Longo, M. R., \& Bertenthal, B. I. (2004). Automaticity and inhibition in action planning. Behavioral and Brain Sciences, 27, 44-45.

Longo, M. R., Kosobud, A., \& Bertenthal, B. I. (2006). Automatic imitation of biomechanically impossible actions: Effects of priming movements vs. goals. Manuscript submitted for publication.

Marcovitch, S., \& Zelazo, P. D. (1999). The A-not-B error: Results from a logistic meta-analysis. Child Development, 70, 1297-1313.

Marcovitch, S., Zelazo, P. D., \& Schmuckler, M. A. (2002). The effect of number of A trials on performance on the A-not-B task. Infancy, 3, 519-529.

Morange, F., \& Bloch, H. (1996). Lateralization of the approach movement and the prehension movement in infants from 4 to 7 months. Early Development and Parenting, 5, 81-92.

Munakata, Y. (1997). Perseverative reaching in infancy: The roles of hidden toys and motor history in the AB task. Infant Behavior and Development, 20, 405-416.

Munakata, Y. (1998). Infant perseveration and implications for object permanence theories: A PDP model of the AB task. Developmental Science, 1, 161-211.

Piaget, J. (1954). The construction of reality in the child (M. Cook, Trans.). New York: Basic Books. (Original work published 1937)

Prinz, W. (1990). A common coding approach to perception and action. In O. Neumann \& W. Prinz (Eds.), Relationships between perception and action (pp. 167-201). Berlin: Springer Verlag.

Provine, R. R., \& Westerman, J. A. (1979). Crossing the midline: Limits of early eye-hand behavior. Child Development, 50, 437-441.

Rizzolatti, G., \& Craighero, L. (2004). The mirror-neuron system. Annual Review of Neuroscience, 27, 169-192.

Rizzolatti, G., Fadiga, L., Gallese, V., \& Fogassi, L. (1996). Premotor cortex and the recognition of motor actions. Cognitive Brain Research, 3, 131-141.

Ruffman, T., \& Langman, L. (2002). Infants' reaching in a multi-well A not B task. Infant Behavior and Development, 25, 237-246.

Schofield, W. N. (1976). Hand movements which cross the body midline: Findings relating age differences to handedness. Perceptual and Motor Skills, 42, 643-646.

Schuberth, R. E., Werner, J. S., \& Lipsitt, L. P. (1978). The stage IV error in Piaget's theory of object concept development: A reconsideration of the spatial localization hypothesis. Child Development, 49, 744-748.

Smith, L. B., Thelen, E., Titzer, R., \& McLin, D. (1999). Knowing in the context of acting: The task dynamics of the A-not-B error. Psychological Review, 106, 235-260.

Sommerville, J. A., \& Woodward, A. L. (2005). Pulling out the intentional structure of action: The relation between action processing and action production in infancy. Cognition, 95, 1-30.

Sommerville, J. A., Woodward, A. L., \& Needham, A. (2005). Action experience alters 3-month-old infants' perception of others' actions. Cognition, 96, B1-B11.

Sophian, C., \& Yengo, L. (1985). Infants' search for visible objects: Implications for the interpretation of early search errors. Journal of Experimental Child Psychology, 40, 260-278.

Spencer, J. P., \& Schutte, A. R. (2004). Unifying representations and responses: Perseverative biases arise from a single behavioral system. Psychological Science, 15, 187-193.

Thelen, E., Schoner, G., Scheier, C., \& Smith, L. B. (2001). The dynamics of embodiment: A field theory of infant perseverative reaching. Behavioral and Brain Sciences, 24, 1-86.

van Hof, P., van der Kamp, J., \& Savelsbergh, G. J. P. (2002). The relation of unimanual and bimanual reaching to crossing the midline. Child Development, 73, 1353-1362.

Wapner, S., \& Cirillo, L. (1968). Imitation of a model's hand movements: Age changes in transposition of left-right relations. Child Development, 39, 887-895. 
Woodward, A. L., \& Sommerville, J. A. (2000). Twelve-month-old infants interpret action in context. Psychological Science, 11, 73-77.

Yates, D. J., \& Bremner, J. G. (1988). Conditions for Piagetian stage IV search errors in a task using transparent occluders. Infant Behavior and Development, 11, 411-417.

Zelazo, P. D., Reznick, J. S., \& Spinazzola, J. (1998). Representational flexibility and response control in a multistep multilocation search task. Developmental Psychology, 34, 203-214. 